\title{
Utilização da espessura de dobras cutâneas para a estimativa da gordura corporal em idosos ${ }^{1}$
}

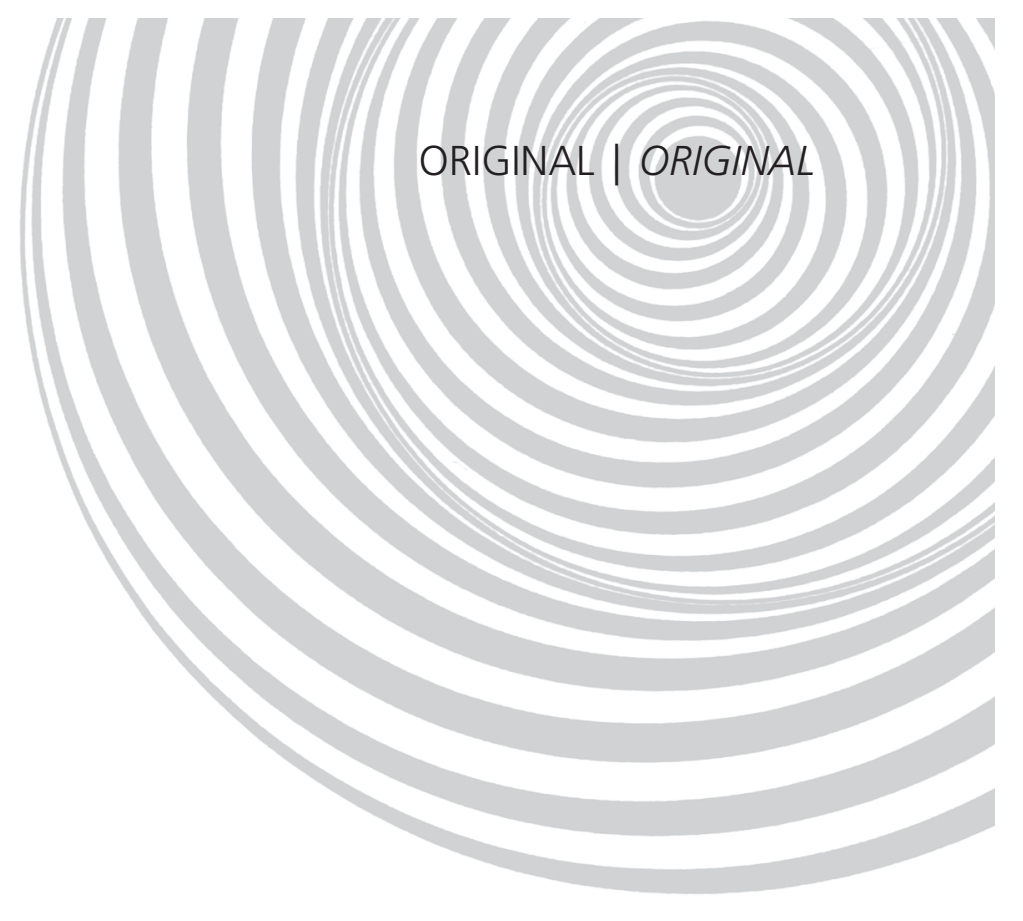

\author{
Estimating body fat from skinfold \\ thicknesses in the elderly
}

Cassiano Ricardo RECH²

Braian Alves CORDEIRO3

Edio Luiz PETROSKI ${ }^{4}$

Francisco de Assis Guedes de VASCONCELOS 5

\section{R E S U M O}

\section{Objetivo}

Analisar a concordância de equações de espessura de dobras cutâneas para a estimativa da gordura corporal em idosos brasileiros, tendo como referência a Absortometria Radiológica de Dupla Energia.

\section{Métodos}

Trata-se de um estudo correlacional. Realizaram-se as medidas antropométricas (estatura, massa corporal, perímetros corporais e espessura de dobras cutâneas) de 180 idosos (60 homens e 120 mulheres) com idades entre 60 e 81 anos. A gordura corporal foi mensurada por meio da Absormetria Radiológica de Dupla Energia e comparada com os valores estimados pelas dobras cutâneas. Foram analisadas as equações de Durnin et al., Jackson et al., Visser et al. e Lean et al. Os critérios de validação cruzada sugeridos por Lohman e a análise gráfica das dispersões em relação à média, por meio do método proposto por Bland \& Altman, foram utilizados.

\section{Resultados}

O grupo avaliado apresentou uma gordura corporal média de 23,1\% ( $D P=5,8 \%$ ) nos homens e 37,3\% ( $D P=6,9 \%)$ nas mulheres, com uma variação entre $6 \%$ a $51,4 \%$. Houve uma elevada concordância entre as equações generalizadas de Durnin et al. e a Absormetria Radiológica de Dupla Energia (64,4\% nos homens e $60,1 \%$ nas mulheres). Nos homens as equações de dobras cutâneas tenderam a superestimar os valores de gordura corporal, já nas mulheres esses valores foram subestimados.

1 Artigo elaborado a partir da dissertação de C.R.RECH, intitulada "Validação de equações antropométricas e de impedância bioelétrica para a estimativa da composição corporal em idosos". Universidade Federal de Santa Catarina; 2006.

2 Universidade Estadual de Ponta Grossa, Programa Pós-Graduação em Educação Física. Av. Ge Carlos Cavalcanti, 4748, Pça Santos Andrade, 84030-900, Ponta Grossa, PR, Brasil. Correspondência para/Correspondence to: C.R.RECH. E-mail: <crrech@hotmail.com>.

3 Universidade do Vale do Itajaí, Departamento de Nutrição. Itajaí, SC, Brasil.

${ }^{4}$ Universidade Federal de Santa Catarina, Programa de Pós-Graduação em Educação Física. Florianópolis, SC, Brasil.

5 Universidade Federal de Santa Catarina, Programa de Pós-Graduação em Nutrição. Florianópolis, SC, Brasil. 
18 | C.R. RECH et al.

\section{Conclusão}

As equações generalizadas desenvolvidas por Durnin et al. são válidas para a estimativa da gordura corporal em idosos do sul do Brasil.

Termos de indexação: Absortometria radiológica de dupla energia. Dobras cutâneas. Gordura corporal. Idoso. Obesidade.

\section{A B S T R A C T}

\section{Objective}

The objective of this study was to analyze skinfold thickness equations for the estimation of body fat in terms of their concordance with dual energy X-ray absorptiometry results in elderly Brazilians.

\section{Methods}

This was a correlation study. Anthropometric measurements (height, body mass, body circumferences and skin fold thicknesses) of 180 elderly subjects (60 men and 120 women) aged 60 to 81 years were taken. Their body fat was measured with dual energy x-ray absorptiometry and compared with the values estimated from skinfold thickness measurements. Equations published by Durnin et al., Jackson et al., Visser et al. and Lean et al. were analyzed. The validation criteria described by Lohman were used together with the Bland and Altman method for graphical analysis of differences with relation to means.

\section{Results}

The men in the study group had a mean body fat of $23.1 \%$ (SD=5.8\%) while the women had a mean body fat of $37.3 \%(S D=6.9 \%)$, with a $6 \%$ to $51.4 \%$ variation. The general equations published by Durnin et al. had great concordance with dual energy $x$-ray absorptiometry (64.4\% for men and $60.1 \%$ for women). Skinfold thickness equations tended to overestimate men's body fat and underestimate women's body fat.

\section{Conclusion}

The general equations proposed by Durnin et al. are a valid tool for estimating body fat in the elderly population of Southern Brazil.

Indexing terms: Dual energy X-ray absorptiometry. Skinfolds thickness. Body fat. Aged. Obesity.

\section{N T R O D U Ç Ã O}

As estimativas dos componentes corporais representam um importante indicador do estado de saúde geral, assim como permitem inferir sobre o estado nutricional de indivíduos e populações. Na população de idosos, em especial, as mudanças na composição corporal servem para monitorar as alterações que ocorrem em função do enveIhecimento. Evidências científicas apontam que ocorre um aumento progressivo na Gordura Corporal $(G C)^{1}$, redução na Massa Corporal Magra $(\mathrm{MCM})^{2}$, além de modificações na quantidade de minerais da $\mathrm{MCM}^{3}$ e na quantidade de água corporal intra e extracelular ${ }^{4}$ com o envelhecimento.

A importância em determinar os componentes corporais reside na estreita relação do aumento da GC, assim como da sua distribuição, com a incidência de doenças cardiovasculares e metabólicas, como diabetes, hipercolesterolemia, hipertensão, entre outras ${ }^{5}$. Além disso, a determinação em estabelecer a quantidade de MCM é de fundamental importância, uma vez que a mesma tem sido relatada como um fator de proteção ao acometimento de distúrbios musculares e quedas em idosos ${ }^{6}$.

As técnicas mais utilizadas para a estimativa da gordura corporal estabelecem relação entre dois importantes componentes: a GC e a MCM, de acordo com o modelo bicomportamental?. Porém, esse modelo é limitado para utilização em idosos, devido às alterações na quantidade da MCM, especialmente na redução da densidade mineral óssea e das alterações na hidratação dos tecidos magros 2,4 . 
A medida da espessura de Dobras Cutâneas (DC) é um método prático e amplamente utilizado para estimar a GC e a MCM. Esta técnica baseia-se na teoria segundo a qual a medida do tecido adiposo de determinados pontos anatômicos do corpo, pode estimar a gordura corporal total, com acuracidade ${ }^{8}$. Entretanto, em idosos algumas limitações, principalmente, relacionadas com a possível redistribuição da gordura subcutânea podem interfir na acuracidade deste método .

Outra técnica que vem se tornando popular na avaliação da composição corporal é a Absortometria Radiológica de Dupla Energia (DEXA), que permite a estimativa da composição corporal (massa gorda, massa livre de gordura e mineral). Esta técnica de estimativa tem sido uma das recomendadas em idosos por requerer pouca colaboração do avaliado, ser confortável e apresentar resultados válidos ${ }^{10,11}$.

Deste modo, o propósito deste estudo é analisar a concordância entre as estimativas de equações de espessura de dobras cutâneas em idosos brasileiros, tendo como método de referência a DEXA.

\section{MÉTODOS}

Os dados analisados são provenientes do banco de dados originário da pesquisa "Validação de equações antropométricas e de impedância bioelétrica para a estimativa da composição corporal em idosos ${ }^{12 "}$, coletados no período entre maio e julho de 2005.

O estudo foi realizado com 180 idosos saudáveis (60 homens e 120 mulheres), com idades entre 60 e 81 anos, todos residentes no município de Florianópolis, Estado de Santa Catarina, localizado na Região Sul do Brasil.

Os indivíduos foram selecionados por convite entre os participantes de quatro grupos de convivência de idosos. Estes grupos foram selecionados devido ao fato de estarem localizados próximos aos locais onde foram realizadas as mensurações, evitando assim maiores transtornos para os idosos.

Foram contactados, por meio de entrevista por telefone, 266 idosos (115 homens e 151 mulheres). Destes, 86 (57 homens e 29 mulheres) não atenderam os critérios de inclusão na amostra, por apresentarem um quadro de pressão arterial alta, possuírem algum tipo de câncer, amputação, marcapasso, idade inferior a 60 anos, ingestão de medicamentos diuréticos, prótese fixa, ou não ter interesse em participar do estudo.

Neste contato por telefone, além dos critérios de inclusão, os idosos responderam um questionário sócio-demográfico e foram agendadas as datas das mensurações antropométricas e da absortometria radiológica de dupla energia.

Esta pesquisa seguiu os princípios éticos de respeito à autonomia das pessoas e o projeto foi aprovado pelo Comitê de Ética em Pesquisa com Seres Humanos da Universidade Federal de Santa Catarina (Protocolo nº 059/05).

As variáveis de massa corporal e estatura foram mensuradas de acordo com a padronização internacional13, Utilizou-se uma balança ARJA, com capacidade para $150 \mathrm{~kg}$ e um estadiômetro de parede com precisão de $0,1 \mathrm{~cm}$. Os perímetros corporais do abdômen, cintura e antebraço foram mensurados por meio de uma fita métrica inelástica, seguindo a padronização de Callaway et al. ${ }^{14}$. A espessura de dobras cutâneas foi mensurada de acordo com a padronização de Harrison et al. ${ }^{15}$ para as dobras cutâneas tricipital, bicipital, subescapular, supra-ilíaca, peitoral, abdominal e coxa medial. Mensuraram-se três medidas adotando-se a média como valor final e todas as medidas foram realizadas por um mesmo avaliador.

A partir das medidas de DC, essas variáveis foram utilizadas em equações de regressão (Tabela 1), em conjunto com as demais medidas antropométricas, para a estimativa da densidade corporal (D - $\mathrm{g} / \mathrm{mL}$ ) ou em percentual de gordura corporal, sendo comparada com a medida-critério (DEXA).

A mensuração da medida critério da GC foi obtida por meio de um scaner de corpo inteiro, 
Tabela 1. Equações de espessura de dobras cutâneas.

\begin{tabular}{|c|c|}
\hline Autores & Equação \\
\hline \multicolumn{2}{|r|}{ Homens } \\
\hline Durnin et al. $18^{*}$ & $D=1,1765-0,0744 \log _{10}\left(X_{1}\right)$ \\
\hline Durnin et al..$^{18^{*}}$ & $D=1,1715-0,0779 \log _{10}\left(X_{1}\right)$ \\
\hline Jackson et al..$^{21}$ & $\begin{array}{l}D=1,0990750-0,0008209\left(X_{2}\right)+0,0000026(X 2)^{2}-0,0002017 \text { (Idade, anos) }-0,00005675 \\
(\text { PABD })+0,00018586(\text { PANT) }\end{array}$ \\
\hline Visser et al..26 & $D=0,0186(1 \mp)-0,0300 \log \left(X_{3}\right)+1,0481$ \\
\hline Lean et al. ${ }^{27}$ & $\% \mathrm{G}=0,353(\mathrm{PCIN})+0,756(\mathrm{TR})+0,235$ (Idade, anos) $-26,4$ \\
\hline \multicolumn{2}{|r|}{ Mulheres } \\
\hline Durnin et $a l . .^{18^{\star *}}$ & $D=1,1567-0,0717 \log _{10}\left(X_{1}\right)$ \\
\hline Durnin et al..$^{18^{*}}$ & $D=1,1339-0,0645 \log _{10}\left(X_{1}\right)$ \\
\hline Jackson et al. ${ }^{21}$ & $D=1,0994921-0,0009929\left(X_{4}\right)+0,0000023\left(X_{4}\right)^{2}-0,0001392$ (Idade, anos) \\
\hline Visser et al..26 & $D=0,0186(0 \mp)-0,0300 \log \left(X_{3}\right)+1,0481$ \\
\hline Lean et al.27 & $\% \mathrm{G}=0,232(\mathrm{PCIN})+0,657(\mathrm{TR})+0,215(\mathrm{ID})-5,5$ \\
\hline
\end{tabular}

* equação específica para idosos; $\mp$ sexo masculino (1) e feminino (0). ** equação generalizada para uma ampla faixa etária. \%G: percentual de gordura corporal; PABD: perímetro abdômen; PANT: P antebraço; PCIN: P cintura; TR: tricipital; D (g/mL): densidade corporal. $\mathrm{X}_{1}: \Sigma$ (tricipital, subescapular, bicipital, suprailíaca); $\mathrm{X}_{2}: \Sigma$ (peitoral, abdominal, coxa média); $\mathrm{X}_{3}: \Sigma$ (bicipital, tricipital); $\mathrm{X}_{4}: \Sigma$ (tricipital, suprailíaca, coxa média).

utilizando-se um aparelho de marca Lunar Prodigy DF +14319 Radiation (Madison, WI), sendo que a quantificação desses valores foi realizada com um software versão 7.52.002 DPX-L.

Os idosos usaram apenas um avental, estiveram descalços, não portavam brincos, anéis, próteses dentárias e outros tipos de materiais metálicos. Foi solicitado ao idoso deitar-se na mesa em decúbito dorsal. O idoso permaneceu imóvel durante a realização do scan, que levou em média 20 a 30 minutos. O aparelho foi calibrado diariamente, de acordo com as recomendações do fabricante. As avaliações foram realizadas por uma técnica em radiologia médica, junto ao Centro de Diagnóstico por Imagens (SONITEC), na cidade de Florianópolis (SC), no período da tarde, no mesmo dia da coleta das medidas antropométricas.

Utilizou-se a análise descritiva das variáveis antropométricas e, para a análise da concordância das equações de DC, foram adotados os procedimentos sugeridos por Lohman ${ }^{16}$. O teste $t$, para amostras pareadas, foi usado para comparação entre as médias estimadas (equações) e mensuradas pela DEXA. Foi utilizada, também, a análise do coeficiente de correlação linear de Pearson ( $r$ ); a análise dos desvios-padrão; a análise do Erro
Constante (EC), que representa a diferença entre os valores estimados e mensurados; a análise do Erro Total $(E T)$, que corresponde à variação dos valores em função do número de sujeitos analisados; e a análise do Erro Padrão de Estimativa (EPE), que representa o erro esperado para a equação analisada em relação à medida-critério (DEXA).

Por fim, foi utilizada a análise de Bland \& Altman ${ }^{17}$, para verificar a dispersão dos escores residuais em função das médias estimadas e mensuradas. As equações de DC foram consideradas válidas, quando atenderam aos critérios acima, ou seja, quando não apresentaram médias estatisticamente diferentes; quando apresentarem $r>0,79$; e valores de EC, ET e EPE inferiores a $3,5 \%$, tanto em homens quanto em mulheres. Utilizou-se o programa Statistical Package for Social Sciences versão 10.0 para a análise dos dados.

\section{RESULTADOS}

A amostra do estudo constituiu-se de 180 idosos, entre 60 e 81 anos de idade, sendo $66,7 \%$ $(n=120)$ mulheres e $33,3 \%(n=60)$ homens. A 
Média (M) de idade entre os homens foi de 69,1 anos (Desvio-Padrão - $\mathrm{DP}=5,6$ ) e das mulheres 67,2 anos (DP=5,1).

As características físicas do grupo de idosos avaliados, no presente estudo, são apresentadas na Tabela 2; pode-se observar que houve diferença significante $(p<0,05)$ para todas as variáveis antropométricas entre homens e mulheres.

A quantidade de gordura corporal apresentou uma amplitude de 6\%-36,3\% em homens

Tabela 2. Características antropométricas e da composição corporal de idosos do município de Florianópolis (SC). 2006.

\begin{tabular}{|c|c|c|c|c|c|c|}
\hline \multirow{2}{*}{ Variáveis } & \multicolumn{3}{|c|}{ Masculino $(n=60)$} & \multicolumn{3}{|c|}{ Feminino $(n=120)$} \\
\hline & Média & DP & Amplitude & Média & $\mathrm{DP}$ & Amplitude \\
\hline Massa corporal $(\mathrm{kg})$ & 74,2 & $10,6^{*}$ & $57,2-103,0$ & 65,6 & 11,1 & $39,8-107,0$ \\
\hline Estatura $(\mathrm{cm})$ & 167,2 & $7,4^{*}$ & $152,0-185,0$ & 154,5 & 5,7 & $141,0-172,0$ \\
\hline $\mathrm{IMC}\left(\mathrm{kg} / \mathrm{m}^{2}\right)$ & 26,5 & $2,7^{*}$ & $20,8-33,7$ & 27,4 & 3,9 & $18,4-39,3$ \\
\hline P Abdômen (cm) & 94,2 & $7,3^{*}$ & $79,5-116,0$ & 90,1 & 9,7 & $67,0-107,0$ \\
\hline P Cintura $(\mathrm{cm})$ & 91,6 & $6,7^{*}$ & $77,0-109,0$ & 82,8 & 8,4 & $66,5-107,0$ \\
\hline P Antebraço (cm) & 26,7 & $1,8^{*}$ & $23,5-32,0$ & 23,7 & 1,8 & $19,4-28,0$ \\
\hline DC SE (mm) & 25,7 & $8,5^{*}$ & $12,0-47,0$ & 28,1 & 9,8 & $6,5-60,0$ \\
\hline DC TR $(\mathrm{mm})$ & 11,7 & $3,2^{*}$ & $5,0-19,5$ & 25,5 & 7,2 & $10,0-47,0$ \\
\hline $\mathrm{DC} \mathrm{BI}(\mathrm{mm})$ & 8,1 & $3,4^{*}$ & $4,0-18,0$ & 17,6 & 6,1 & $5,9-40,0$ \\
\hline DC PT (mm) & 24,1 & $7,1^{*}$ & $7,0-35,2$ & 23,4 & 6,1 & $6,5-36,3$ \\
\hline DC SI (mm) & 22,8 & $6,9^{*}$ & $10,8-45,0$ & 28,4 & 8,8 & $8,1-46,2$ \\
\hline $\mathrm{DC} \mathrm{ABD}(\mathrm{mm})$ & 33,6 & $8,3^{*}$ & $12,0-55,0$ & 42,3 & 10,4 & $10,0-63,2$ \\
\hline $\mathrm{DC} C X(\mathrm{~mm})$ & 15,9 & $5,3^{*}$ & $8,0-29,0$ & 35,3 & 11,2 & $10,5-61,0$ \\
\hline$\% G C_{D E X A}$ & 23,1 & $5,8^{*}$ & $6,0-36,3$ & 37,3 & 6,9 & $15,6-51,4$ \\
\hline $\mathrm{MLG}_{\mathrm{DEXA}}(\mathrm{kg})$ & 54,9 & $6,8^{*}$ & $42,5-70,6$ & 38,9 & 4,5 & $26,1-52,5$ \\
\hline
\end{tabular}

* diferenças estatísticas entre os sexos, teste $t$ para amostras independentes $(p<0,05)$.

IMC: índice de massa corporal; P: perímetro corporal; DC: espessura de dobra cutânea; SE: subescapular; TR: tricipital; BI: bicipital; PT: peitoral; SI: supra-ilíaca; ABD: abdominal vertical; CX: coxa medial; \%GC: percentual de gordura corporal; MLG: massa livre de gordura; DP: desvio-padrão.

Tabela 3. Análise dos critérios de concordância (validação cruzada) das equações de espessura de dobras cutâneas para a estimativa do percentual de gordura corporal em idosos do município de Florianópolis (SC), 2006.

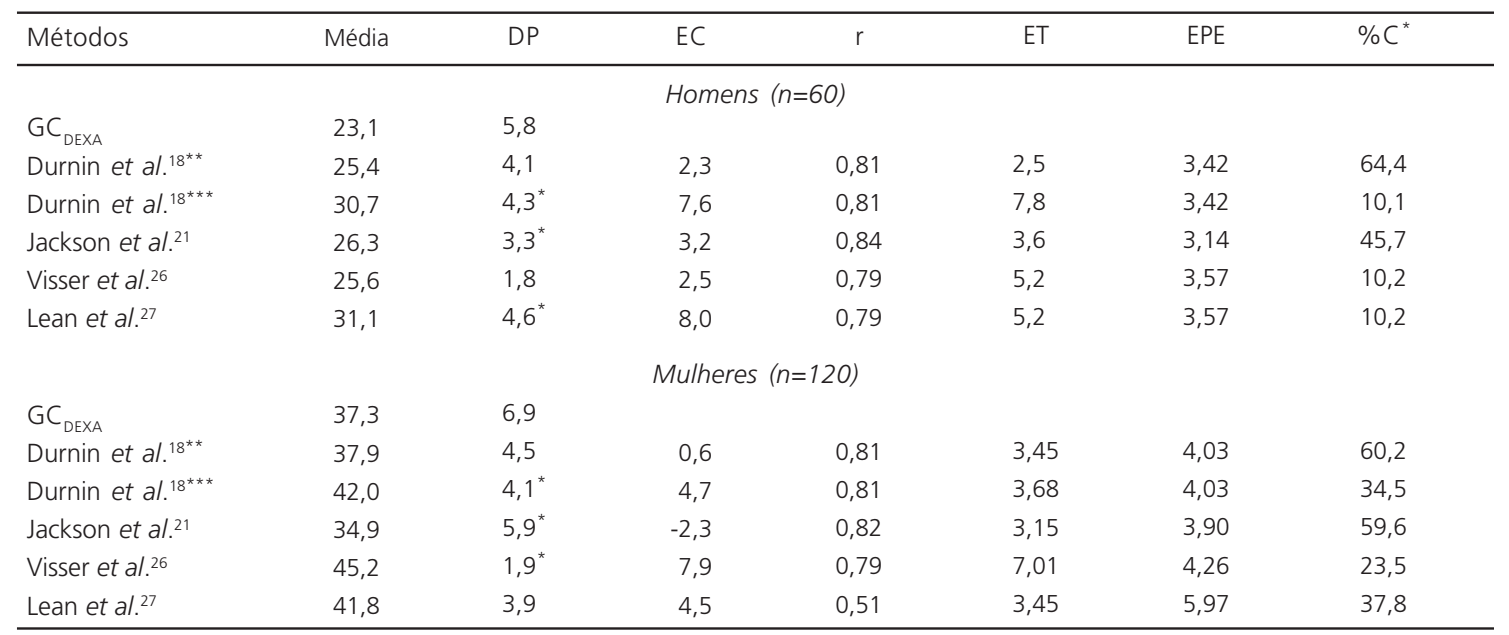

*\%C: limite de concordância ou validação $(3,5 \%) ;{ }^{* *}$ equação generalizada para uma ampla faixa etária; ${ }^{* *}$ equação específica para idosos; difere significantemente $(p<0,05)$ teste $t$ pareado.

EC: erro constante; ET: erro total; EPE: erro padrão de estimativa; $\mathrm{GC}_{\mathrm{DEXA}}$ : gordura corporal medida pelo métdo absortometria radiológica de dupla energia; DP: desvio-padrão. 
e 15,6\%-51,4\% em mulheres, demonstrando em mulheres um valor estatisticamente maior do que nos homens $(p<0,05)$. Já a MLG, avaliada por meio da DEXA, foi maior nos homens $(54,9$, $D P=6,8 \mathrm{~kg})$ que nas mulheres $(38,9, D P=4,5 \mathrm{~kg})$.
A Tabela 3 apresenta os valores médios, desvios e os critérios de concordância (validação cruzada) para as equações analisadas. Nota-se que para os homens a equação generalizada de Durnin et al..$^{18}$ não diferiu em relação à medida
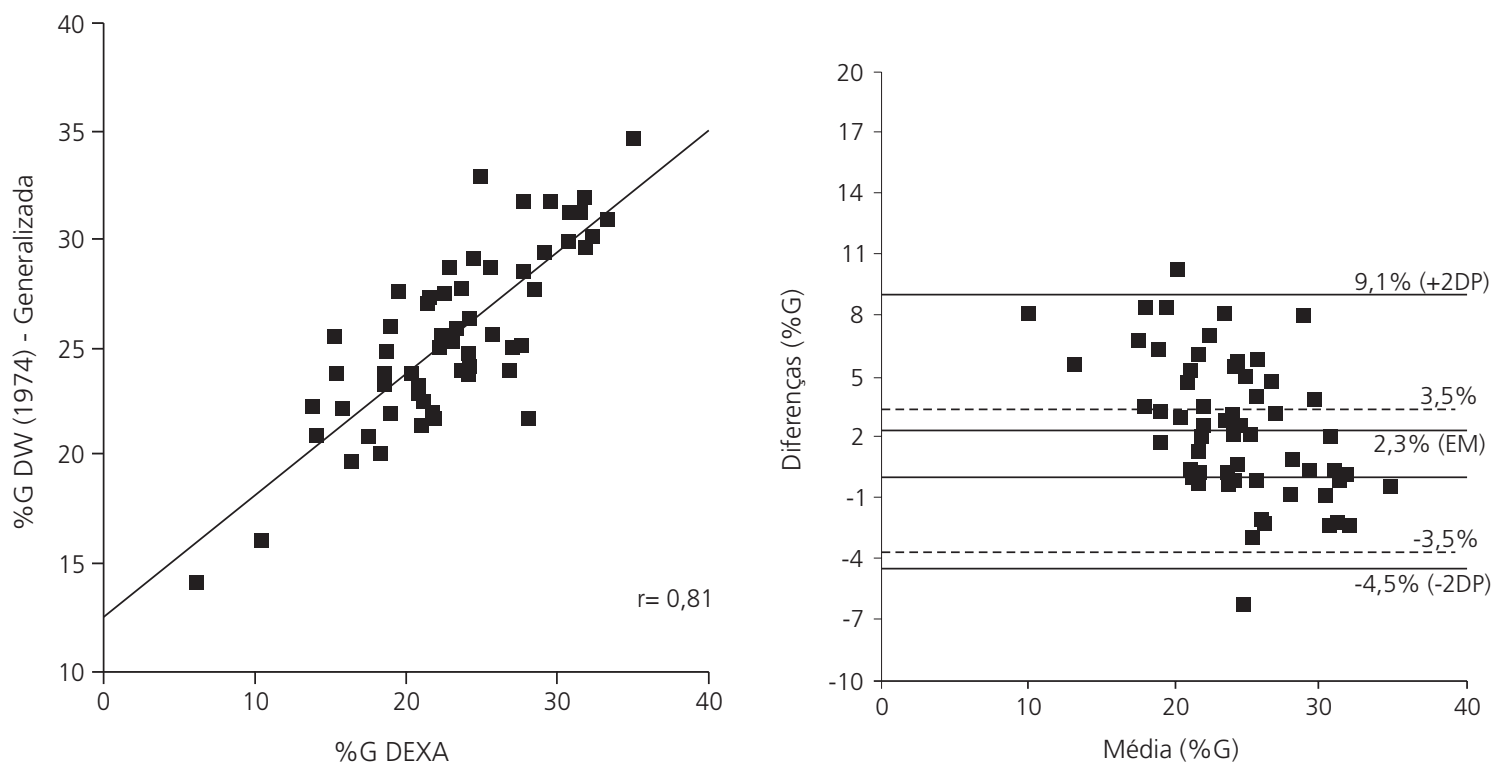

Figura 1. Dispersão entre os métodos da absortometria radiológica de dupla energia e da equação generalizada de Durnin et al. ${ }^{18}$ para estimativa da gordura corporal em homens idosos. Florianópolis (SC), 2006.
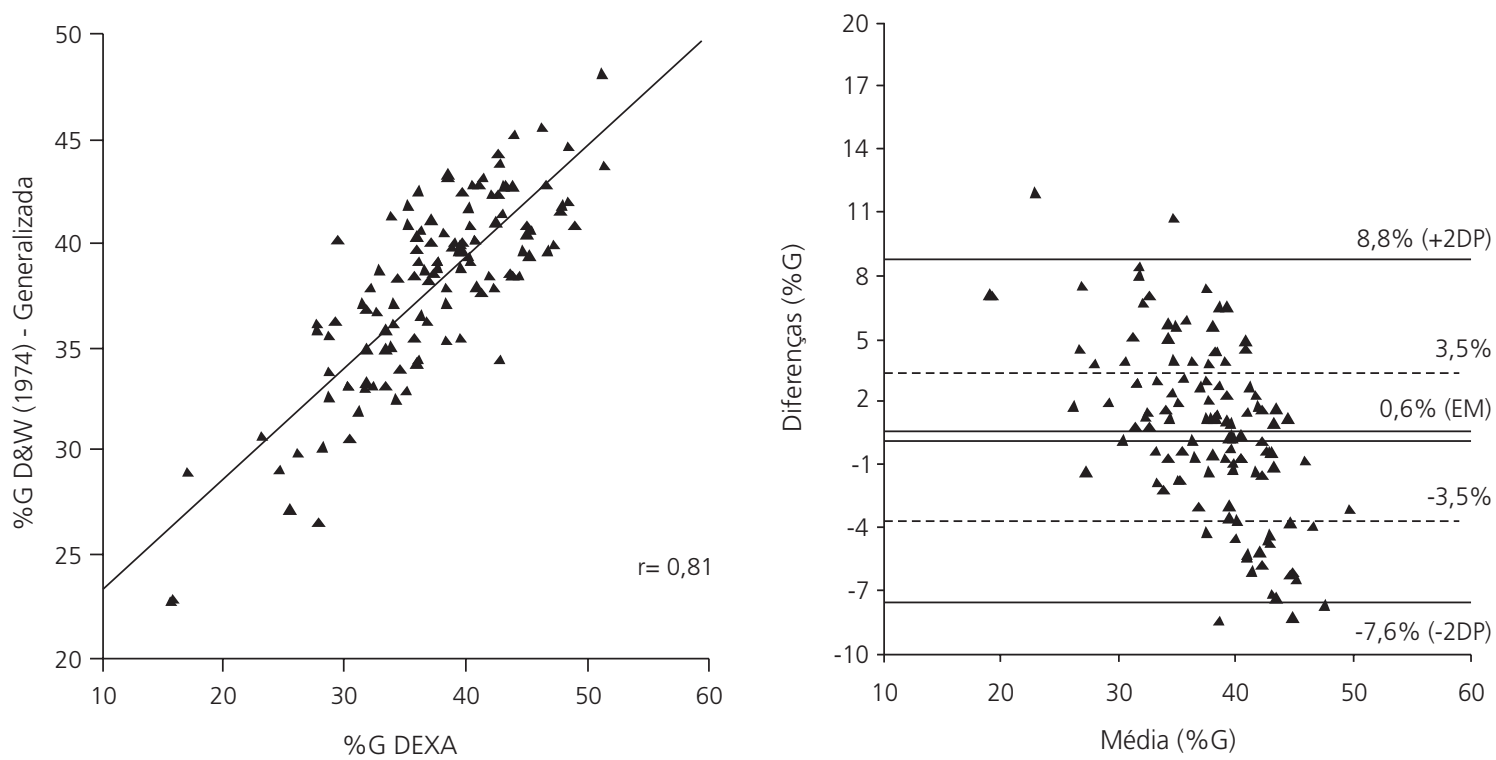

Figura 2. Dispersão entre os métodos da absortometria radiológica de dupla energia e da equação generalizada de Durnin et al. ${ }^{18}$ para estimativa da gordura corporal em mulheres idosas. Florianópolis (SC), 2006. 
da DEXA, apresentando um erro constante (EC) de 2,3\% GC. Nas mulheres as equações generalizadas de Durnin et al. ${ }^{18}$ não apresentaram diferenças significantes $(p<0,05)$, tendo um EC de $0,6 \% \mathrm{GC}$.

As Figuras 1 e 2 apresentam o coeficiente de correlação linear de Pearson e a análise da dispersão dos escores em relação ao erro médio. As equações de Durnin et al. ${ }^{18}$ apresentaram valores de correlação significantes, demonstrando uma linearidade entre os escores dos dois métodos testados.

\section{I S C U S S Ã O}

A medida da espessura de dobras cutâneas têm sido umas das técnicas mais utilizadas para a estimativa da gordura corporal em estudos populacionais. A excelente relação entre o somatório da espessura de dobras cutâneas e a quantidade de gordura corporal total é um dos principais fatores para que esse método seja considerado um método válido para a estimativa da GC em seres humanos ${ }^{8}$.

Heymsfield et al. ${ }^{8}$ analisaram a relação da soma da espessura de quatro dobras cutâneas (bicipital, tricipital, subescapular e supra-ilíaca) com a quantidade de gordura total, por meio da técnica da ressonância magnética e relataram uma forte relação $(r=0,90)$ e um baixo erro $(4,8 \mathrm{~kg})$.

Baseado em estudos que estabeleceram essas evidências da relação entre a GC e o somatório da espessura de dobras cutâneas foram desenvolvidas diversas equações que atendessem às características dos diferentes grupos etários e étnicos ${ }^{19,20}$. No presente estudo 10 equações foram analisadas em comparação com a medida da DEXA na população de idosos.

As equações específicas para idosos desenvolvidas por Durnin et al. ${ }^{18}$ apresentaram uma tendência de superestimar os valores de GC, tanto em homens quanto em mulheres, sendo que a concordância entre os métodos foi baixa (entre $10,1 \%$ e $34,5 \%)$.
O número reduzido de idosos mensurados no desenvolvimento da equação no estudo original (24 homens e 37 mulheres), assim como as diferentes médias de GC entre os grupos de idosos de Durnin et al..$^{18}$ e os do presente estudo, podem explicar parte desta variação nas diferenças. Outra questão observada foi que nas mulheres idosas a amplitude dos valores de GC apresentada por Durnin et al. ${ }^{18}(26 \%-52 \%)$ e a do presente estudo (15,6\%-51,4\%) é diferente, demonstrando assim uma tendência de superestimar a GC, em idosas com GC abaixo de $26 \%$.

Essa equação já havia apresentado diferenças estatísticas quando testada em 15 mulheres pós-menopausa, tendo superestimado em 7,2\% a GC em relação à medida da DEXA ${ }^{21}$. Esses resultados confirmam também os achados de Barbosa et al. ${ }^{11}$, que, em 20 mulheres idosas, encontraram uma superestimativa de $4 \%$ na GC em comparação com medida da DEXA.

Diferentemente disso, a equação generalizada de Durnin et al. ${ }^{18}$, proposta por meio de uma amostra de 481 indivíduos entre 17-72 anos de idade, mostrou-se válida para os idosos no presente estudo (Tabela 3). Também foi observada uma alta concordância entre os métodos da DEXA e as equações generalizadas de Durnin et al. ${ }^{18}$, variando entre $60,2 \%$ a $64,4 \%$. Os coeficientes de correlação desta equação (Figuras 1 e 2), tanto em homens quanto em mulheres, foram considerados válidos ${ }^{18}$.

Pode-se notar que nos homens o Erro Médio (EM) foi maior (2,3\%) do que o apresentado nas mulheres $(0,6 \%)$ e a dispersão foi menor $(13,6 \%)$ em relação às mulheres $(16,4 \%)$, porém as duas são consideradas elevadas (Intervalo de Confiança - IC $=95 \%$ ).

O fato de apresentar um maior número de indivíduos mensurados e um modelo logarítmico pode ter contribuído para a melhor adequação dos resultados da equação generalizada em comparação com os resultados obtidos pela equação específica de Durnin et al. ${ }^{18}$.

As equações generalizadas, desenvolvidas por Jackson et al. ${ }^{22}$ para homens e Jackson et al. ${ }^{23}$ para mulheres, também foram analisadas neste 
estudo. Essas equações não são específicas para o grupo de idosos, porém, devido ao fato de ter sido mensurado um grupo de idosos jovens, de terem sido desenvolvidas com um $n$ amostral considerável e por ter sido utilizado um modelo quadrático para o desenvolvimento, essas equações foram testadas no grupo de idosos.

Os modelos quadráticos são mais rígidos em relação aos métodos de regressão linear, pois levam em consideração uma maior variabilidade dos valores extremos, podendo, assim, considerar a variabilidade ocasionada pelo envelhecimento ${ }^{24}$.

Apesar disso, essas equações apresentaram uma estimativa de GC diferente do apresentado pela DEXA, tanto em homens quanto em mulheres $(p<0,05)$, com erros entre $-4,1 \%$ e $3,6 \%$. Houve uma tendência de subestimação do \% G nas muIheres e superestimativa nos homens.

A concordância entre os métodos foi baixa, variando entre $45,7 \%$ (homens) e 59,6\% (mulheres), mesmo tendo apresentado um coeficiente de correlação alto, $r=0,84$ para os homens e $r=0,82$ para as mulheres. Os valores de correlação foram próximos dos apresentados na validação cruzada em 95 homens e 82 mulheres, em que foram observados valores de $r=0,91$ (homens) e $r=0,82$ (mulheres). Rech et al. ${ }^{25}$, comparando as medidas de GC da DEXA com os escores obtidos pela equação de Jackson et al. ${ }^{23}$ para 15 mulheres, observaram um erro-médio de 1,24\%, porém a concordância ( $D P=3,5 \% G C)$ foi de apenas $46,6 \%$ da amostra analisada.

Outro estudo que se propôs a analisar a validade da equação de Jackson et al. ${ }^{22}$, foi elaborado por Barbosa et al. ${ }^{11}$, que observaram que esta equação superestimou em 9,7\% a GC em 20 mulheres idosas, quando comparada com as medidas da DEXA.

Algumas considerações são necessárias para analisar as equações propostas por Jackson et al. ${ }^{22}$ e Jackson et al. ${ }^{23}$, pois as mesmas vêm sendo amplamente utilizadas em estudos no Brasil. Outra equação desenvolvida pelos mesmos autores, utilizando o somatório da espessura de sete dobras cutâneas, mostrou-se válida para ser utilizada em indivíduos adultos no Brasi ${ }^{26}$. No entanto, em idosos, essa equação, que utiliza sete dobras cutâneas não foi testada, devido à pouca praticidade em mensurar sete dobras cutâneas para estimar a GC.

Até mesmo o fato de algumas DC apresentarem elevados valores de erros inter e intra-avaliador pode contribuir para elevar os erros nas estimativas de $G^{21}$. Outro fato que pode ter contribuído para a não concordância (validade) desta equação é a utilização de um adipômetro diferente do utilizado no estudo original, quando foi utilizado um equipamento Lange, enquanto no presente estudo utilizou-se um aparelho Cescorf digital.

A influência do tipo de adipômetro na medida da espessura de dobras cutâneas é relatada por Cyrino et al. ${ }^{21}$, que, comparando as médias de espessura de DC em nove diferentes pontos, encontraram diferenças estatísticas em todos os pontos, quando compararam os valores obtidos pelos adipômetros Lange e Cescorf.

Além da equação generalizada de Durnin et al. ${ }^{18}$, a equação de Visser et al. ${ }^{26}$, baseada na medida do somatório da espessura das dobras cutâneas bicipital e tricipital, apresentou valores médios que não diferem estatisticamente em relação à medida-critério, para o sexo masculino. Porém, notou-se uma forte tendência de superestimativa dos valores de GC, quando comparado com a DEXA, sendo que $49,2 \%$ da amostra apresentaram uma concordância de 3,5\% GC. Além disso, o EPE foi elevado (3,66\%). Nas mulheres, a equação de Visser et al. ${ }^{26}$ também superestimou a GC entre $6,4 \%$ e $7,9 \%$, erros que diferem estatisticamente $(p<0,05)$ da medida da DEXA; a concordância foi menor $(27,7 \%)$ do que a observada nos homens.

O desvio-padrão desta equação, em ambos os sexos, foi inferior ao apresentado pelo método da DEXA, demonstrando uma tendência à homogeneidade dos dados em torno da média, ou seja, os valores extremos de GC tendem a ser próximos da média. Esse é um dos critérios sugeridos por Lohman ${ }^{16}$ para a análise da validade de equações antropométricas. Assim, o ET foi muito elevado, demonstrando que essas equações não são ade- 
quadas para serem utilizadas na população de idosos nacionais.

Por fim, a última equação analisada neste estudo foi proposta por Lean et al. ${ }^{27}$, baseada nas medidas de perímetros da cintura, da dobra cutânea tricipital, além da idade e do sexo. Quando analisados os critérios de validação (Tabela 3), pode-se observar que a mesma superestimou a GC em homens e mulheres. O coeficiente de correlação foi forte $(r=0,79)$ nos homens e moderado nas mulheres $(r=0,51)$, no entanto, houve uma maior concordância nas estimativas das mulheres $(37,8 \%)$ do que na dos homens (10,2\%). Mesmo assim, os valores de EPE e Erro Total (ET) são considerados inadequados para utilização em idosos.

Alguns fatores, como a experiência do avaliador, equipamento, padronização, localização do ponto anatômico e pinçamento da espessura da dobra cutânea, representam erros consideráveis na avaliação da composição corporal por meio do método de espessura de $D C^{18}$.

Além disso, em sujeitos com mais idade, devido ao envelhecimento e à redistribuição da gordura corporal, evidencia-se uma tendência de maior acúmulo da gordura interna em relação à gordura subcutânea, assim como ocorre uma migração dos depósitos de gordura dos membros para o tronco ${ }^{9,28}$. Isso auxilia para que a relação entre as medidas subcutâneas não represente de forma real a medida de gordura corporal tota ${ }^{28}$.

Também a diminuição da compressibilidade da espessura da dobra cutânea pode interferir significativamente na medida ${ }^{18}$. As diferenças no padrão de distribuição de gordura corporal entre diferentes etnias podem alterar a relação entre o somatório da espessura de DC e a medida da composição corporal, assim como valores de densidade corporal ${ }^{29}$. Por exemplo, em uma investigação conduzida com indivíduos Mexicanos ${ }^{30}$ desenvolveu uma equação antropométrica para estimativa da massa gorda utilizando a espessura das dobras cutâneas bicipital e panturrilha média, ajustadas por sexo e por massa corporal $(r 2=0,85$; $E P E=3,2 \mathrm{~kg}$ ). Assim, nota-se que esses fatores podem contribuir para erros na avaliação da composição corporal de pessoas com mais idade em diferentes etnias, por meio da utilização do método de espessura de dobras cutâneas. Assim, as mensurações devem seguir as padronizações estabelecidas, usar equipamentos adequados e calibrados e ser realizadas por avaliadores experientes para diminuir essas variações.

Com base nos resultados é possível sugerir que as equações generalizadas desenvolvidas por Durnin et al. ${ }^{18}$, com base na espessura de dobras cutâneas, são adequadas para a estimativa da gordura corporal em idosos. Desse modo, estudos que necessitem a estimativa da gordura corporal em idosos podem utilizar a espessura de dobras cutâneas.

\section{COLABORADORES}

C.R. RECH e B.A. CORDEIRO foram responsáveis pela coleta, pela análise dos dados e pela redação do artigo. E.L. PETROSKI e F.A.G. VASCONCELOS participaram do desenho metodológico, da análise dos dados e da revisão do artigo.

\section{A GRADECIMENTOS}

Ao Centro de Diagnóstico por Imagens e à Fundação de Apoio à Pesquisa Científica e Tecnológica do Estado de Santa Catarina, pelo apoio financeiro ao projeto de pesquisa.

\section{REFERÊ NCIAS}

1. Horani MH, Mooradian AD. Management of obesity in the elderly: special considerations. Treat Endocrinol. 2002; 1(6):387-98.

2. Nassis GP, Geldas ND. Age-related pattern in body composition changes for 18-69 year old women. J Sports Med Phys Fitness. 2003; 43(3):327-33.

3. Topinková E. Aging, disability and fralty. Ann Nutr Metab. 2008; 52(Suppl 1):6s-11s.

4. Chumlea WC, Guo SS, Zeller CM, Reo NV, Baumgartner RN, Garry PJ, et al. Total body water reference values and prediction equations for adults. Kidney Int. 2001; 59(6):2250-58. doi: 10.1046/j.1523-1755.2001.00741.x.

5. Allison DB, Fontaine KR, Manson JE, Stevens J, Vanltallie TB. Annual deaths attributable to obesity in the United States. JAMA. 1999; 282(16):1530-38. 
6. Janssen I, Baumgartner RN, Ross R, Rosenberg IH, Roubenoff R. Skeletal muscle cutpoints associated with elevated physical disability risk in older men and women. Am J Epidemiol. 2004;159(4):413-21. doi: 10.1093/aje/kwh058.

7. Siri WE. Body composition from fluid spaces and density: analysis of methods. In: Brozeck J, Henschel A, editors. Techniques for measuring body composition. Washington (DC): National Academy of Science; 1961.

8. Heymsfield SD, Nunez C, Testolin C, Gallagher D. Anthropometry and methods of body composition measurement for research and field application in the elderly. Eur J Clin Nutr. 2000; 54(3):26-32.

9. Hughes VA, Roubenoff R, Wood M, Frontera WR, Evans WJ, Fiatarone MA. Anthropometric assessment of 10-y changes in body composition in the elderly. Am J Clin Nutr. 2004; 80(2):475-82.

10. Ball SD, Swan PD. Accuracy of estimating intraabdominal fat in obese women. Exerc Physiol. 2003; 6(4):1121-6.

11. Barbosa AB, Santarém JM, Jacob Filho W, Meirelles ES, Marucci MFN. Comparação da gordura corporal de mulheres idosas segundo antropometria, bioimpedância e DEXA. Arch Latinoam Nutr. 2001; 51(1):125-35.

12. Rech CR. Validação de equações antropométrica e de impedância bioelétrica para a estimativa da composição corporal em idosos [dissertação]. Florianópolis: Universidade Federal de Santa Catarina; 2006.

13. Gordon CC, Chumlea WC, Roche AF. Stature, recumbent length, weight. In: Lohman TG, Roche AF, Martorell R, editors. Anthropometric standardizing reference manual. Champaign (Illinois): Human Kinetics Books; 1991. p.3-8.

14. Callaway CW, Chumlea WC, Bouchard C, Himes $J H$, Lohman TG, Martin AD, et al. Circumferences. In: Lohman TG, Roche AF, Martorell R, editors. Anthropometric standardizing reference manual. Champaign (Illinois): Human Kinetics Books; 1991. p.39-54.

15. Harrison GG, Buskirk ER, Carter JEL, Johnston FE, Lohman TG, Pollock ML, et al. Skinfold thicknesses and measurements technique. In: Lohman TG, Roche AF, Martorell R, editors. Anthropometric standardizing reference manual. Champaign (Illinois): Human Kinetics Books; 1991. p.55-80.

16. Lohman TG. Advances in body composition assessment. Champaign (Illinois): Human Kinetics Publishers; 1992.

17. Bland JM, Altman DG. Statistical methods for assessing agreement between two methods of clinical measurements. Lancet. 1986; 1(8476): 307-10.

18. Durnin JV, Womersley J. Body fat assessed from body density and its estimation from skinfold thickness: measurement on 481 men and women aged from 16 at 72 years. Br J Nutr. 1974; 32(1): 77-97.

19. Heyward VH. Pratical body composition assessment for children, adults, children, adults, and older adults. Int J Sport Nutr. 1998; 8(3):285-307.

20. Kuriyan R, Thomas T, Kurpad AV. Total body muscle mass estimation from bioelectrical impedance analysis and simple anthropometric measurements in Indian men. Indian J Med Res. 2008;127(5): 441-6. doi: 10.1080/03014469700005102.

21. Cyrino ES, Okano AH, Glaner MF, Romanzini $M$, Gobbo LA, Makoski A, et al. Impacto da utilização de diferentes compassos de dobras cutâneas para a análise da composição corporal. Rev Bras Med Esporte. 2003; 9(3):21-8. doi: 10.1590/\$151786 922009000700009.

22. Jackson AS, Pollock ML. Generalized equations for predicting body density of men. Br J Nutr. 1978; 40(3):497-502.

23. Jackson AS, Pollock ML, Ward A. Generalized equations for prediction body density of women. Med Sci Sports Exerc. 1980; 12(3):175-82.

24. Vincent WJ. Statiscs in kinesiology. California: California State University; 1995.

25. Rech CR, Silva AT, Lunardi CC, Bohrer T, Petroski EL. Comparação da absortometria radiológica de dupla energia, antropometria e bioimpedância na avaliação da composição corporal em mulheres. Rev Eletr EFDEPortes. 2005; 10(90):1-5.

26. Visser M, Heuvel EVD, Deurenberg P. Prediction for the estimation of body composition in the elderly using anthropometric data. Br J Nutr. 1994; 71(6): 823-33.

27. Lean MEJ, Han TS, Deurenberg P. Prediction body composition by densitometry from simple anthropometric measurements. Am J Clin Nutr. 1996; 63(1):4-14.

28. Micozzi MS, Harris TM. Age variations in the relation of body indices to estimates of body composition fat and muscle mass. Am J Phys Anthropol. 1990; 81(3):375-9.

29. Mott JW, Wang J, Thornton JC, Allison DB, Heymsfield SB, Pierson RN. Relation between body fat and age in 4 ethnic groups. Am J Clin Nutr. 1999; 69(5):1007-13.

30. Huerta RH, Esparza-Romero J, Urquidez R, Pacheco BI, Valencia ME, Alemán-Mateo H. Validez de una ecuación basada en antropometría para estimar la grasa corporal en adultos mayores. Arch Latinoam Nutr. 2007; 57(4):357-65.

Recebido em: 20/4/2008

Versão final reapresentada em: 1/10/2008 Aprovado em: 16/6/2009 